\title{
Biology and Life Table Studies on Healthy and Pasteuria penetrans Infected Meloidogyne incognita
}

\author{
S. Prabhu*, J. Sandeep Kumar ${ }^{2}$ and R. Poorniammal ${ }^{3}$ \\ ${ }^{1}$ Department of Plant Protection, Horticultural College and Research Institute, \\ Periyakulam, India \\ ${ }^{2}$ Department of Entomology, Agricultural College and Research Institute, \\ Madurai, India \\ ${ }^{3}$ Department of Natural Resource Management, Horticultural College and Research Institute, \\ Periyakulam, India \\ *Corresponding author
}

\section{A B S T R A C T}

\section{Keywords}

Pasteuria penetrans, Meloidogyne incognita,

\section{Article Info}

Accepted:

25 February 2020

Available Online:

10 March 2020
Pasteuria penetrans is an important parasite of Meloidogyne incognita. Lifecycle of this parasite is in close association with the nematode. Life table was constructed for healthy and $P$. penetrans infected population of $M$. incognita. Results revealed that the life cycle of $P$. penetrans infected $M$. incognita was extend by 3 days when compared to healthy population. Survival and life expectancy of the infected population was reduced by 2 days. Stable age distribution revealed that reduction in survival of infected population in all stages. Fecundity rate was reduced to four folds compared to healthy population. This subsequently reduced the population doubling time.

\section{Introduction}

A global agricultural lose of $\$ 157$ billion was annually incurred due to plant parasitic nematodes (Abad et al., 2008). Root knot nematode Meloidogyne incognita is an economically important parasite. Life table is a systematic study of survival and mortality of a population. It is used to determine whether a population is growing, declaning or remaining stale. It is highly useful in studying factors responsible for change in population size (Atwal and bains 1974).

It is used to determine whether a population is growing declining or remaining stable. It gives a knowledge on rate of development of an organism, age specific fecundity. This stimulates the outcome of management decessions. Many authors had developed life 
table studies for many nematodes viz. $M$. arenaria (Ferris and Hunt 1979) Heterodeara cajani (Singh and Sharma 1995). The present study attempted to compare the life table of healthy and Pasteruia penetrans infected $M$. incognita population on tomato.

\section{Materials and Methods}

Seeds of tomato cv.Co3 were sown in a seedling pan. After germination the seedlings of equal height were transplanted to 80 tumbler pots (40 for infected and 40 for healthy nematodes) of $7 \mathrm{~cm}$ diameter containing steam sterilized soil. The seedlings were allowed to establish for 10 days. $P$. penetrans encumbered $\mathrm{J} 2$ were prepared by adding fresh spores extracted from white females. Healthy J2 were centrifuged along with spores of $P$. penetrans in $1.5 \mathrm{ml}$ eppendorf tubes at 500rpm for $2 \mathrm{~min}$. The nematodes were examined for 100 per cent encumbrance under steriozoom microscope. Freshly hatched healthy and $P$. penetrans infected juveniles at cohort were inoculated at the rate of $400 \mathrm{~J} 2$ per pot. Nematode development and age specific survival were studied by removing the seedlings at every 24 hours after inoculation. The roots were stained with acid fuchsin-lactophenol and destined in plain lactophenol. The roots were compressed between two glass plates $(15 \times 10$ $\mathrm{cm})$ and the number of juveniles in different stage was counted under a stereo zoom microscope. If the internal body content of the nematode is not intact, they were considered as dead nematodes. Peak occurrence of each life stage was used to calculate survival and development of different stages. The procedure was continued till adult females were developed. All data were corrected and adjusted in such a way that the life table commences with a cohort of $100 \mathrm{~J} 2$ as described by Singh and Sharma (1994). Life table was constructed as described by Deevey (1947). The fecundity and intrinsic rate of natural increase of population were calculated by using Birch (1948) formula as elaborated by Howe (1953). The following columns were used.

\begin{tabular}{|c|c|}
\hline $\mathrm{X}$ & Pivotal age days \\
\hline $\mathrm{l}_{\mathrm{x}}$ & Age specific longevity \\
\hline $\mathrm{m}_{\mathrm{x}}$ & Age specific fecundity \\
\hline $\mathrm{Ro}\left(\mathrm{l}_{\mathrm{x}} \mathrm{m}_{\mathrm{x}}\right)$ & Net reproductive rate \\
\hline $\mathrm{rm}=\ln \mathrm{R}_{\mathrm{o}} / \mathrm{T}_{\mathrm{c}}$ & $\begin{array}{c}\text { Innate capacity for increase of females per female } \\
\text { per day) }\end{array}$ \\
\hline $\mathrm{X}\left(\mathrm{d}_{\mathrm{x}}\right)$ & Number of dying individual in cohort \\
\hline $1000 \mathrm{q}_{\mathrm{x}}=\mathrm{d}_{\mathrm{x}} / \mathrm{l}_{\mathrm{x}} * 100$ & Per cent apparent mortality \\
\hline $100 \mathrm{dx} / \mathrm{n}$ & Per cent real mortality \\
\hline $\mathrm{Lx}=1_{\mathrm{x}}+(\mathrm{x}+1) / 2$ & Number alive between age $\mathrm{x}$ and $\mathrm{x}+1$ \\
\hline $\mathrm{X} \mathrm{T}_{\mathrm{x}}$ & Number of individual life days beyond age \\
\hline $\mathrm{ex}=\mathrm{T}_{\mathrm{x}} / \mathrm{l}_{\mathrm{x}}$ & Expectation of further life days \\
\hline$\sum \mathrm{m}_{\mathrm{x}}$ & Gross Reproductive Rate $(\mathrm{GRR})$ \\
\hline $\mathrm{R}_{\mathrm{o}}=\sum_{\mathrm{x}} \mathrm{l}_{\mathrm{x}} \mathrm{m} \mathrm{m}_{\mathrm{x}}$ & Net Reproductive Rate (NRR) \\
\hline $\mathrm{Tc}=\left(\sum \mathrm{l}_{\mathrm{x}} \mathrm{m}_{\mathrm{x}}\right) / \mathrm{R}_{\mathrm{o}}$ & Mean Length of Generation (MLG) \\
\hline $\mathrm{Rc}=\left(\log _{\mathrm{Ro}}\right) / \mathrm{T}_{\mathrm{c}}$ & Innate capacity for increase in number $)$ \\
\hline $\mathrm{T}=\left(\operatorname{lnR}_{\mathrm{o}}\right) / \mathrm{rm}$ & Corrected generation time in days \\
\hline $\mathrm{Rm}=\mathrm{e}^{7-\mathrm{rm}} \cdot 1_{\mathrm{x}} \mathrm{m}_{\mathrm{x}}$ & Intrinsic rate of increase \\
\hline $\mathrm{T}=\left(\log _{\mathrm{e}} \cdot \mathrm{R}_{\mathrm{o}}\right) / \mathrm{rm}$ & Corrected generation time days \\
\hline$\Lambda=\mathrm{e}^{\mathrm{rm}}$ & Finite rate of increase \\
\hline $\mathrm{C}=\left(\mathrm{e}^{-\mathrm{rmxlx}}\right) / \sum \mathrm{e}^{-\mathrm{rmxlx}}$ & Stable age distribution \\
\hline
\end{tabular}




\section{Results and Discussion}

\section{Biology of M. incognita}

The biology of spore encumbered $\mathrm{J} 2$ was extended by 3 days compared to healthy $\mathrm{J} 2$. The results revealed that survival of $\mathbf{J} 2$ was increased by $P$. penetrans infection. The spore burden on the $\mathrm{J} 2$ make them difficult to penetrate the host and only $\mathrm{J} 2$ which have few spore reached the host. J2 with heavy spores attachment survived till the food reserves in them are depleted (Stirling, 1984).

Competition and other factors might weaken the $\mathrm{J} 2$ and predispose them to infection by microorganisms (Davies et.al., 1988; Davies, 2009). Infected J2 which successfully reached the host became J3. The duration of infected J3 was extended by one day compared to healthy J3. Subsequently this led to increase in the duration of $\mathrm{J} 4$ by one day. Oviposition period was also increased by 2 days. Increase in life cycle of $P$. penetrans infected $M$. incognita lead to increase in total life cycle by 3 days. Further the bacterium reduced the fecundity of $M$. incognita by 5 fold compared to healthy female populations. However, $P$. penetrans was not found to cause any morphological changes in the host. (Davies et al., 2011) (Table 1).

Survival and life expectancy of $M$. incognita infected by $P$. penetrans

The study on life table provides a concise summary of certain vital population statistics (Brich, 1948). According to Southwood (1976) it is the most useful numerical aid in studying population biology enabling determination of age distribution and mortality in natural populations of any organisms. Life table gives a vivid picture on the fecundity and growth potential of any organism under prevailing environmental conditions.

Table.1 Biology of healthy and $P$. penetrans infected $M$. incognita

\begin{tabular}{|c|c|c|c|c|}
\hline \multirow[t]{2}{*}{ Parameters } & \multicolumn{2}{|c|}{$\begin{array}{l}\text { Duration of stages in } \\
\text { healthy nematodes }\end{array}$} & \multicolumn{2}{|c|}{$\begin{array}{l}\text { Duration of stages in } \\
\text { infected nematodes }\end{array}$} \\
\hline & $\begin{array}{l}\text { Range } \\
\text { (Days) }\end{array}$ & $\overline{\mathbf{X}} \pm \mathrm{SE}$ & $\begin{array}{l}\text { Range } \\
\text { (Days) }\end{array}$ & $\overline{\mathbf{X}} \pm \mathrm{SE}$ \\
\hline Incubation period & $3-5$ & $4.5 \pm 0.57$ & $3-5$ & $4.6 \pm 0.50$ \\
\hline $\mathbf{J}_{2}$ & $4-5$ & $4.7 \pm 0.1$ & $8-10$ & $9.2 \pm 0.20$ \\
\hline $\mathbf{J}_{\mathbf{3}}$ & $4-5$ & $4.4 \pm 0.1$ & $5-6$ & $5.5 \pm 0.33$ \\
\hline $\mathbf{J}_{\mathbf{4}}$ & $3-4$ & $3.7 \pm 0.1$ & $4-5$ & $4.3 \pm 0.21$ \\
\hline Preovipositional period & $1-3$ & $2.4 \pm 0.57$ & $1-3$ & $2.7 \pm 0.60$ \\
\hline Oviposition period & $10-14$ & $12.3 \pm 1.15$ & $12-15$ & $14.3 \pm 0.88$ \\
\hline $\begin{array}{l}\text { Total development period } \\
\text { (egg - adult) }\end{array}$ & $25-36$ & $30 \pm 1.40$ & $34-44$ & $39 \pm 1.34$ \\
\hline Fecundity & $270-300$ & $286 \pm 8.81$ & $78-83$ & $80.3 \pm 3.26$ \\
\hline $\begin{array}{l}\text { Average temperature } 28 \pm 5^{\circ} \mathrm{C} \\
\text { Average RH } 70 \pm 5 \% \\
\pm \text { represents SE of mean }\end{array}$ & & & & \\
\hline
\end{tabular}


Table.2 Survival and life expectancy of $M$. incognita infected with $P$. penetrans

\begin{tabular}{|c|c|c|c|c|c|c|c|}
\hline $\begin{array}{l}\text { Age in day } \\
(X)\end{array}$ & $\begin{array}{c}\text { Numbers } \\
\text { surviving } \\
\text { in } X\left(l_{x}\right)\end{array}$ & $\begin{array}{l}\text { Numbers } \\
\text { dying in } X \\
\left(d_{x}\right)\end{array}$ & $\begin{array}{c}\text { Per cent } \\
\text { apparent } \\
\text { mortality } \\
100 \mathbf{q} x=d_{x} l_{x} * 100\end{array}$ & $\begin{array}{l}\text { Per cent real } \\
\text { mortality } \\
100 d_{x} / n\end{array}$ & $\begin{array}{c}\text { No. alive } \\
\text { between age } \\
x \text { and } X+1 \\
L_{x}=I_{x}+(X \\
+1) / 2\end{array}$ & $\begin{array}{l}\text { No. of individual } \\
\text { life days beyond } \\
\text { age } X\left(T_{x}\right)\end{array}$ & $\begin{array}{c}\text { Expectation } \\
\text { of further } \\
\text { life days } e_{x}= \\
T_{x} / I_{x}\end{array}$ \\
\hline 0 & 100 & 0 & 0.00 & 0.00 & 99 & 2326 & 23.26 \\
\hline 1 & 98 & 2 & 2.04 & 2.00 & 95.5 & 2227 & 22.72 \\
\hline 2 & 93 & 5 & 5.38 & 5.00 & 93 & 2131.5 & 22.92 \\
\hline 3 & 93 & 0 & 0.00 & 0.00 & 92.5 & 2038.5 & 21.92 \\
\hline Egg & 93 & 7 & 7.62 & 7.00 & - & - & - \\
\hline 4 & 92 & 1 & 1.09 & 1.00 & 90.5 & 1946 & 21.15 \\
\hline 6 & 85 & 4 & 4.71 & 4.00 & 84 & 1768.5 & 20.81 \\
\hline 8 & 78 & 5 & 6.41 & 5.00 & 78 & 1604 & 20.56 \\
\hline 10 & 74 & 4 & 5.41 & 4.00 & 74 & 1450 & 19.59 \\
\hline $\mathbf{J}_{2}$ & 74 & 19 & 23.40 & 19.00 & - & - & - \\
\hline 12 & 72 & 2 & 2.78 & 2.00 & 70 & 1303 & 18.10 \\
\hline 14 & 68 & 0 & 0.00 & 0.00 & 68 & 1165 & 17.13 \\
\hline 16 & 68 & 0 & 0.00 & 0.00 & 68 & 1029 & 15.13 \\
\hline $\mathbf{J}_{3}$ & 68 & 6 & 8.66 & 9.00 & - & - & - \\
\hline 17 & 68 & 0 & 0.00 & 0.00 & 68 & 961 & 14.13 \\
\hline 18 & 68 & 0 & 0.00 & 0.00 & 68 & 893 & 13.13 \\
\hline 19 & 68 & 0 & 0.00 & 0.00 & 68 & 825 & 12.13 \\
\hline 20 & 68 & 0 & 0.00 & 0.00 & 68 & 757 & 11.13 \\
\hline $\mathbf{J}_{4}$ & 68 & $\mathbf{0}$ & $\mathbf{0}$ & 0.00 & - & - & - \\
\hline 21 & 68 & 0 & 0.00 & 0.00 & 68 & 689 & 10.13 \\
\hline 22 & 68 & 0 & 0.00 & 0.00 & 68 & 621 & 9.13 \\
\hline 23 & 68 & 0 & 0.00 & 0.00 & 68 & 553 & 8.13 \\
\hline 24 & 68 & 0 & 0.00 & 0.00 & 68 & 485 & 7.13 \\
\hline 25 & 68 & 0 & 0.00 & 0.00 & 68 & 417 & 6.13 \\
\hline 26 & 68 & 0 & 0.00 & 0.00 & 64 & 349 & 5.13 \\
\hline 27 & 60 & 8 & 13.33 & 8.00 & 58 & 285 & 4.75 \\
\hline 28 & 56 & 4 & 7.14 & 4.00 & 52.5 & 227 & 4.05 \\
\hline 29 & 49 & 7 & 14.29 & 7.00 & 45.5 & 174.5 & 3.56 \\
\hline 30 & 42 & 7 & 16.67 & 7.00 & 36 & 129 & 3.07 \\
\hline 31 & 30 & 12 & 40.00 & 12.00 & 28.5 & 93 & 3.10 \\
\hline 32 & 27 & 3 & 11.11 & 3.00 & 24 & 64.5 & 2.39 \\
\hline 33 & 21 & 6 & 28.57 & 6.00 & 18 & 40.5 & 1.93 \\
\hline 34 & 15 & 6 & 40.00 & 6.00 & 15 & 22.5 & 1.50 \\
\hline 35 & 15 & 0 & 0.00 & 0.00 & 7.5 & 7.5 & 0.50 \\
\hline Adult & 0 & 15 & 0.00 & 15.00 & 0 & 0 & 0.00 \\
\hline
\end{tabular}


Table.3 Survival and life expectancy of healthy M. incognita on tomato

\begin{tabular}{|c|c|c|c|c|c|c|c|}
\hline $\begin{array}{l}\text { Age in } \\
\text { day }(X)\end{array}$ & $\begin{array}{l}\text { Number } \\
\quad \text { S } \\
\text { survivin } \\
\text { g in X } \\
\left(\mathbf{l}_{\mathbf{x}}\right)\end{array}$ & $\begin{array}{c}\text { Number } \\
\text { s dying } \\
\text { in } X\left(d_{x}\right)\end{array}$ & $\begin{array}{c}\text { Per cent } \\
\text { apparent } \\
\text { mortality } \\
100 q x=d_{x} l_{x} \\
* 100\end{array}$ & $\begin{array}{l}\text { Per cent } \\
\text { real } \\
\text { mortality } \\
\operatorname{100d}_{x} / \mathbf{n}\end{array}$ & $\begin{array}{c}\text { No. alive } \\
\text { between } \\
\text { age } x \text { and } \\
X+1 L_{x}= \\
l_{x}+(X+1) / \\
2\end{array}$ & $\begin{array}{c}\text { No. of } \\
\text { individual } \\
\text { life days } \\
\text { beyond age } \\
\text { X T }_{\mathbf{x}}\end{array}$ & Expectation of further life days $e_{x}=T_{x} / l_{x}$ \\
\hline 0 & 100 & 0 & 0.00 & 0.00 & 99.5 & 2516 & 25.16 \\
\hline 1 & 99 & 1 & 1.01 & 1.00 & 95.5 & 2416.5 & 24.41 \\
\hline 2 & 92 & 7 & 7.61 & 7.00 & 92 & 2321 & 24.23 \\
\hline 3 & 92 & 0 & 0.00 & 0.00 & 92 & 2229 & 24.23 \\
\hline Egg & 92 & 8 & 8.62 & 8.00 & - & - & - \\
\hline 4 & 92 & 0 & 0.00 & 0.00 & 91 & 2137 & 23.23 \\
\hline 5 & 90 & 2 & 2.22 & 2.00 & 89 & 2046 & 22.73 \\
\hline 6 & 88 & 2 & 2.27 & 2.00 & 88 & 1957 & 22.24 \\
\hline 7 & 88 & 0 & 0.00 & 0.00 & 86.5 & 1869 & 21.24 \\
\hline 8 & 85 & 3 & 3.53 & 3.00 & 85 & 1782.5 & 20.97 \\
\hline 9 & 85 & 0 & 0.00 & 0.00 & 85 & 1697.5 & 19.97 \\
\hline $\mathbf{J}_{2}$ & 85 & 7 & 8.02 & 7.00 & - & - & - \\
\hline 10 & 85 & 0 & 0.00 & 0.00 & 84.5 & 1612.5 & 18.97 \\
\hline 11 & 84 & 1 & 1.19 & 1.00 & 84 & 1528 & 18.19 \\
\hline 12 & 84 & 0 & 0.00 & 0.00 & 84 & 1444 & 17.19 \\
\hline 13 & 84 & 0 & 0.00 & 0.00 & 83 & 1360 & 16.19 \\
\hline $\mathbf{J}_{3}$ & 84 & 1 & 1.19 & 1.00 & - & - & - \\
\hline 14 & 82 & 0 & 0.00 & 0.00 & 82 & 1277 & 15.57 \\
\hline 15 & 82 & 0 & 0.00 & 0.00 & 82 & 1195 & 14.57 \\
\hline 16 & 82 & 0 & 0.00 & 0.00 & 82 & 1113 & 13.57 \\
\hline 17 & 82 & 0 & 0.00 & 0.00 & 82 & 1031 & 12.57 \\
\hline $\mathbf{J}_{4}$ & 82 & $\mathbf{0}$ & 0 & 0.00 & - & - & - \\
\hline 18 & 82 & 0 & 0.00 & 0.00 & 82 & 949 & 11.57 \\
\hline 19 & 82 & 0 & 0.00 & 0.00 & 82 & 867 & 10.57 \\
\hline 20 & 82 & 0 & 0.00 & 0.00 & 81 & 785 & 9.57 \\
\hline 21 & 80 & 2 & 2.50 & 2.00 & 80 & 704 & 8.80 \\
\hline 22 & 80 & 0 & 0.00 & 0.00 & 80 & 624 & 7.80 \\
\hline 23 & 80 & 0 & 0.00 & 0.00 & 80 & 544 & 6.80 \\
\hline 24 & 80 & 0 & 0.00 & 0.00 & 80 & 464 & 5.80 \\
\hline 25 & 80 & 0 & 0.00 & 0.00 & 80 & 384 & 4.80 \\
\hline 26 & 80 & 0 & 0.00 & 0.00 & 75 & 304 & 3.80 \\
\hline 27 & 70 & 10 & 14.29 & 10.00 & 65 & 229 & 3.27 \\
\hline 28 & 60 & 10 & 16.67 & 10.00 & 57 & 164 & 2.73 \\
\hline 29 & 54 & 6 & 11.11 & 6.00 & 48 & 107 & 1.98 \\
\hline 30 & 42 & 12 & 28.57 & 12.00 & 40 & 59 & 1.40 \\
\hline 31 & 38 & 4 & 10.53 & 4.00 & 19 & 19 & 0.50 \\
\hline Adult & 0 & 38 & 0.00 & 38.00 & 0 & 0 & \\
\hline
\end{tabular}


Table.4 Stable age distribution of Meloidogyne incognita juveniles infected by $P$. penetrans

\begin{tabular}{|c|c|c|c|c|c|c|c|}
\hline Age & $I_{x}$ & $\mathbf{m}_{\mathbf{x}}$ & $l_{x} m_{x}$ & $\mathbf{X} \cdot \mathbf{I}_{\mathrm{x}} \cdot \mathbf{m}_{\mathrm{x}}$ & $\mathrm{e}^{-\mathrm{rmx}} \cdot \mathbf{l}_{\mathrm{x}}$ & $\begin{array}{l}\text { Stable age } \\
\text { distribution }\end{array}$ & $\begin{array}{l}\text { Percentage } \\
\text { distribution }\end{array}$ \\
\hline 1 & 1 & & & & 0.8353 & 0.186281 & \\
\hline 2 & 0.98 & & & & 0.6837 & 0.152484 & \\
\hline 3 & 0.93 & & & & 0.5420 & 0.120867 & 46 \\
\hline 4 & 0.93 & & & & 0.4527 & 0.100957 & \\
\hline 5 & 0.92 & & & & 0.3740 & 0.083419 & \\
\hline 6 & 0.89 & & & & 0.3022 & 0.067406 & \\
\hline 7 & 0.85 & & & & 0.2411 & 0.053771 & \\
\hline 8 & 0.83 & & & & 0.1967 & 0.043857 & \\
\hline 9 & 0.78 & & & & 0.1544 & 0.034426 & \\
\hline 10 & 0.78 & & & & 0.1289 & 0.028755 & 41 \\
\hline 11 & 0.74 & & & & 0.1022 & 0.022786 & \\
\hline 12 & 0.74 & & & & 0.0853 & 0.019033 & \\
\hline 13 & 0.72 & & & & 0.0694 & 0.015468 & \\
\hline 14 & 0.68 & & & & 0.0547 & 0.012202 & \\
\hline 15 & 0.68 & & & & 0.0457 & 0.010192 & 8 \\
\hline 16 & 0.68 & & & & 0.0382 & 0.008513 & \\
\hline 17 & 0.68 & & & & 0.0319 & 0.007111 & \\
\hline 18 & 0.68 & & & & 0.0266 & 0.005939 & \\
\hline 19 & 0.68 & & & & 0.0222 & 0.004961 & \\
\hline 20 & 0.68 & & & & 0.0186 & 0.004144 & 3 \\
\hline 21 & 0.68 & 5.2 & 3.54 & 74.26 & 0.0155 & 0.003461 & \\
\hline 22 & 0.68 & 6.6 & 4.49 & 98.74 & 0.0130 & 0.002891 & \\
\hline 23 & 0.68 & 6.8 & 4.62 & 106.35 & 0.0108 & 0.002415 & \\
\hline 24 & 0.68 & 7.2 & 4.90 & 117.50 & 0.0090 & 0.002017 & \\
\hline 25 & 0.68 & $\begin{array}{c}10 . \\
1\end{array}$ & 6.87 & 171.70 & 0.0076 & 0.001685 & \\
\hline 26 & 0.68 & $\begin{array}{c}10 . \\
2\end{array}$ & 6.94 & 180.34 & 0.0063 & 0.001407 & \\
\hline 27 & 0.6 & 6.3 & 3.78 & 102.06 & 0.0047 & 0.001037 & \\
\hline 28 & 0.56 & 5.8 & 3.25 & 90.94 & 0.0036 & 0.000809 & \\
\hline 29 & 0.49 & 5.5 & 2.70 & 78.16 & 0.0026 & 0.000591 & \\
\hline 30 & 0.42 & 4.5 & 1.89 & 56.70 & 0.0019 & 0.000423 & \\
\hline 31 & 0.3 & 3.7 & 1.11 & 34.41 & 0.0011 & 0.000252 & \\
\hline 32 & 0.27 & 3.2 & 0.86 & 27.65 & 0.0009 & 0.000190 & \\
\hline 33 & 0.21 & 2.6 & 0.55 & 18.02 & 0.0006 & 0.000123 & \\
\hline 34 & 0.15 & 1.4 & 0.21 & 7.14 & 0.0003 & 0.000074 & \\
\hline 35 & 0.15 & 1.2 & 0.18 & 6.30 & 0.0003 & 0.000061 & \\
\hline 36 & 0 & 0 & 0 & 0 & 0.0000 & 0.000000 & 2 \\
\hline
\end{tabular}


Table.5 Stable age distribution of healthy Meloidogyne incognita juveniles

\begin{tabular}{|c|c|c|c|c|c|c|c|}
\hline Age & $\mathbf{l}_{\mathbf{x}}$ & $\mathbf{m}_{\mathbf{x}}$ & $\mathbf{l}_{\mathbf{x}} \mathbf{m}_{\mathrm{x}}$ & $\mathbf{X} . \mathbf{l}_{\mathbf{x}} \cdot \mathbf{m}_{\mathbf{x}}$ & $\mathrm{e}^{-\mathrm{rmx}} \cdot \mathrm{Ix}$ & $\begin{array}{c}\text { Stable age } \\
\text { distribution }\end{array}$ & $\begin{array}{l}\text { Percentage } \\
\text { distribution }\end{array}$ \\
\hline 1 & 1 & & & & 0.8353 & 0.1804491 & \multirow{3}{*}{45} \\
\hline 2 & 0.99 & & & & 0.6907 & 0.1384722 & \\
\hline 3 & 0.92 & & & & 0.5361 & 0.1074836 & \\
\hline 4 & 0.92 & & & & 0.4478 & 0.0897779 & \multirow[t]{5}{*}{79} \\
\hline 5 & 0.92 & & & & 0.3740 & 0.0749888 & \\
\hline 6 & 0.9 & & & & 0.3056 & 0.0612743 & \\
\hline 7 & 0.88 & & & & 0.2496 & 0.0500432 & \\
\hline 8 & 0.88 & & & & 0.2085 & 0.0417996 & \\
\hline 9 & 0.85 & & & & 0.1682 & 0.0337237 & \multirow[t]{4}{*}{11} \\
\hline 10 & 0.85 & & & & 0.1405 & 0.0281684 & \\
\hline 11 & 0.85 & & & & 0.1174 & 0.0235282 & \\
\hline 12 & 0.84 & & & & 0.0969 & 0.0194212 & \\
\hline 13 & 0.84 & & & & 0.0809 & 0.0162220 & \multirow[t]{4}{*}{6} \\
\hline 14 & 0.84 & & & & 0.0676 & 0.0135497 & \\
\hline 15 & 0.82 & & & & 0.0551 & 0.0110482 & \\
\hline 16 & 0.82 & & & & 0.0460 & 0.0092282 & \\
\hline 17 & 0.82 & & & & 0.0384 & 0.0077081 & \multirow[t]{16}{*}{4} \\
\hline 18 & 0.82 & 22.3 & 18.286 & 329.148 & 0.0321 & 0.0064383 & \\
\hline 19 & 0.82 & 23 & 18.860 & 358.340 & 0.0268 & 0.0053777 & \\
\hline 20 & 0.82 & 26.5 & 21.730 & 434.600 & 0.0224 & 0.0044919 & \\
\hline 21 & 0.8 & 29.3 & 23.440 & 492.240 & 0.0183 & 0.0036604 & \\
\hline 22 & 0.8 & 31.3 & 25.040 & 550.880 & 0.0153 & 0.0030574 & \\
\hline 23 & 0.8 & 25.4 & 20.320 & 467.360 & 0.0127 & 0.0025538 & \\
\hline 24 & 0.8 & 25.4 & 20.320 & 487.680 & 0.0106 & 0.0021331 & \\
\hline 25 & 0.8 & 24.3 & 19.440 & 486.000 & 0.0089 & 0.0017817 & \\
\hline 26 & 0.8 & 21.4 & 17.120 & 445.120 & 0.0074 & 0.0014882 & \\
\hline 27 & 0.7 & 15.2 & 10.640 & 287.280 & 0.0054 & 0.0010877 & \\
\hline 28 & 0.6 & 14.3 & 8.580 & 240.240 & 0.0039 & 0.0007787 & \\
\hline 29 & 0.54 & 14.3 & 7.722 & 223.938 & 0.0029 & 0.0005854 & \\
\hline 30 & 0.42 & 10.2 & 4.284 & 128.520 & 0.0019 & 0.0003803 & \\
\hline 31 & 0.38 & 9.2 & 3.496 & 108.376 & 0.0014 & 0.0002874 & \\
\hline 32 & 0 & 0 & 0.000 & 0.000 & 0.0000 & 0.0000000 & \\
\hline
\end{tabular}

In the present study percent mortality of egg stage ranged from 7 to 8 . Even though the life expectancy of $M$. incognita infected with $P$. penetrans extended by 2 days, mortality rate of J2 was high (19\%) (Table 2) when compared to healthy $\mathrm{J} 2$ (7 \%) (Table 3). These results were in accordance with Bansa - Singh and Dhawan (1994) who showed negative correlation between the number of spores per $\mathrm{J} 2$ and penetration of $\mathrm{J} 2$ into the roots. Encumbered spores reduced the mortality of $\mathrm{J} 2$ and maintained them in active state which provides them a greater chance in contacting the host (Davies et al., 1991). The duration from $\mathrm{J} 2$ to $\mathrm{J} 3$ was increased by one day. Mortality rate of $P$. penetrans infected J3 was high $(9 \%)$ when compared to healthy population (1\%) (Table $2 \& 3$ ). The mortality 
of healthy $\mathrm{J} 2$ was less after reaching the host. This could be due to stable environment compared to complex soil ecosystem where various factors affect the J2 (Singh and Sharma 1994). From J3 to J4 no mortality was recorded. The duration of $P$. penetrans infected and healthy adult stage remained the same. $P$. penetrans infection extends the life cycle of $M$. incognita by 3 days. Survival and life expectancy of $M$. incognita parasitized by $P$. penetrans was reduced by 2 days compared to healthy population.

Stable age distribution of healthy and Pasteuria penetrans infected $M$. incognita

The contribution made by different developmental stages was determined by analyzing the stable age distribution. The study on stable age distribution revealed that per cent distribution of various life stages was higher in healthy $M$. incognita and lowest in $M$. incognita parasitized by $P$. penetrans. The distribution of encumbered population of $\mathrm{J} 2$ was reduced to nearly 41 per cent (Table 4 ) of the healthy population (79 \%) (Table 5). The distribution of $\mathrm{J} 2$ was much affected by $P$. penetrans when compared to other stages. Rangaswamy et al., (2001) reported that the development of $\mathrm{J} 2$ into adult was lower in $P$. penetrans infected nematodes. The per cent survival of infected female decreases with increase in time spent by the $\mathrm{J} 2$ in soil (Giannakou and Gowen, 2004).

Table.8 Population growth statistics of $M$. incognita infected with $P$. penetrans

\begin{tabular}{|c|c|}
\hline Parameters & $\begin{array}{l}\text { M. incognita } \\
\text { infected with } \\
\text { P. penetrans }\end{array}$ \\
\hline Gross reproductive rate $\left(\sum \mathrm{m}_{\mathrm{x}}\right)$ & 80.3 \\
\hline Net reproductive rate $\left(\mathrm{Ro}=\sum \mathrm{l}_{\mathrm{x}} \mathrm{m}_{\mathrm{x}}\right)$ & 39.16 \\
\hline Mean length of generation (days) $\left\{\mathrm{Tc}=\left(\sum \mathrm{l}_{\mathrm{x}} \mathrm{m}_{\mathrm{x}}\right) / \mathrm{R}_{\mathrm{o}}\right\}$ & 25.09 \\
\hline $\begin{array}{l}\text { Innate capacity for increase } \quad \text { (females/female/day) } \\
\mathrm{rm}=\left(\log R_{0}\right) / T_{\mathrm{c}}\end{array}$ & 0.14 \\
\hline $\begin{array}{l}\text { Finite rate of increase in number (females / females / day) } \\
\left(\Lambda=\mathrm{e}^{\mathrm{rm}}\right)\end{array}$ & 1.15 \\
\hline Rate of weekly multiplication of population $\left(\mathrm{e}^{\mathrm{rm}}\right)^{7}$ & 2.74 \\
\hline Doubling time $\left(\log _{2} / \log \lambda\right)$ & 4.80 \\
\hline
\end{tabular}

Table.9 Population growth statistics of healthy M. incognita

\begin{tabular}{|l|c|}
\hline \multicolumn{1}{|c|}{ Parameters } & M. incognita \\
\hline Gross reproductive rate $\left(\sum \mathrm{m}_{\mathrm{x}}\right)$ & 292.1 \\
\hline Net reproductive rate $\left(\mathrm{Ro}=\sum \mathrm{l}_{\mathrm{x}} \mathrm{m}_{\mathrm{x}}\right)$ & 219.27 \\
\hline $\begin{array}{l}\text { Mean length of generation }(\mathrm{days})\left\{\mathrm{T}_{\mathrm{c}}=\left(\sum \mathrm{l}_{\mathrm{x}} \mathrm{m}_{\mathrm{x}}\right) / \mathrm{R}_{\mathrm{o}}\right\} \\
\text { Innate capacity for increase } \quad(\text { females/female/day) }\end{array}$ & 22.98 \\
$\mathrm{rm}=\left(\log \mathrm{R}_{\mathrm{o}}\right) / \mathrm{T}_{\mathrm{c}}$ & 0.23 \\
\hline $\begin{array}{l}\text { Finite rate of increase in number (females / females / day) } \\
\left(\Lambda=\mathrm{e}^{\mathrm{rm}}\right)\end{array}$ & 1.26 \\
\hline Rate of weekly multiplication of population $\left(\mathrm{e}^{\mathrm{rm}}\right)^{7}$ & 5.16 \\
\hline Doubling time $\left(\log _{2} / \log \lambda\right)$ & 2.95 \\
\hline
\end{tabular}




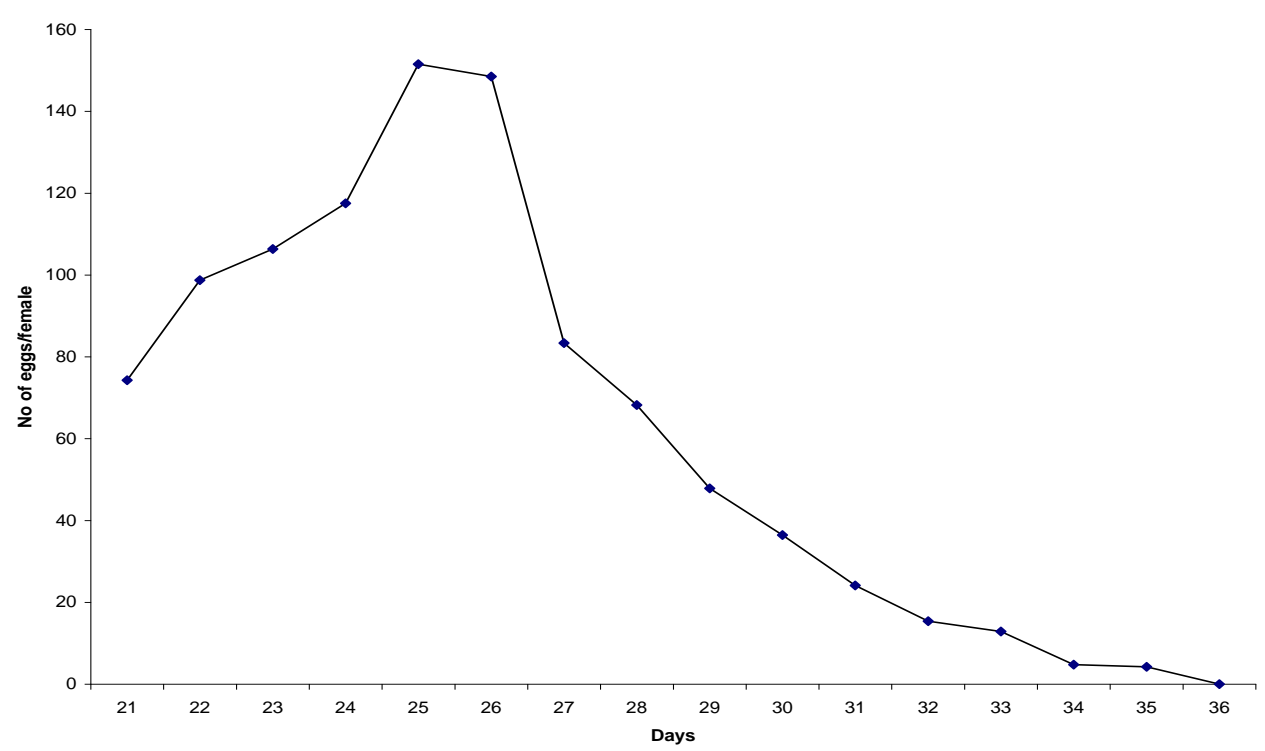

Fig.1 Age specific fecundity for females infected with P. penetrans

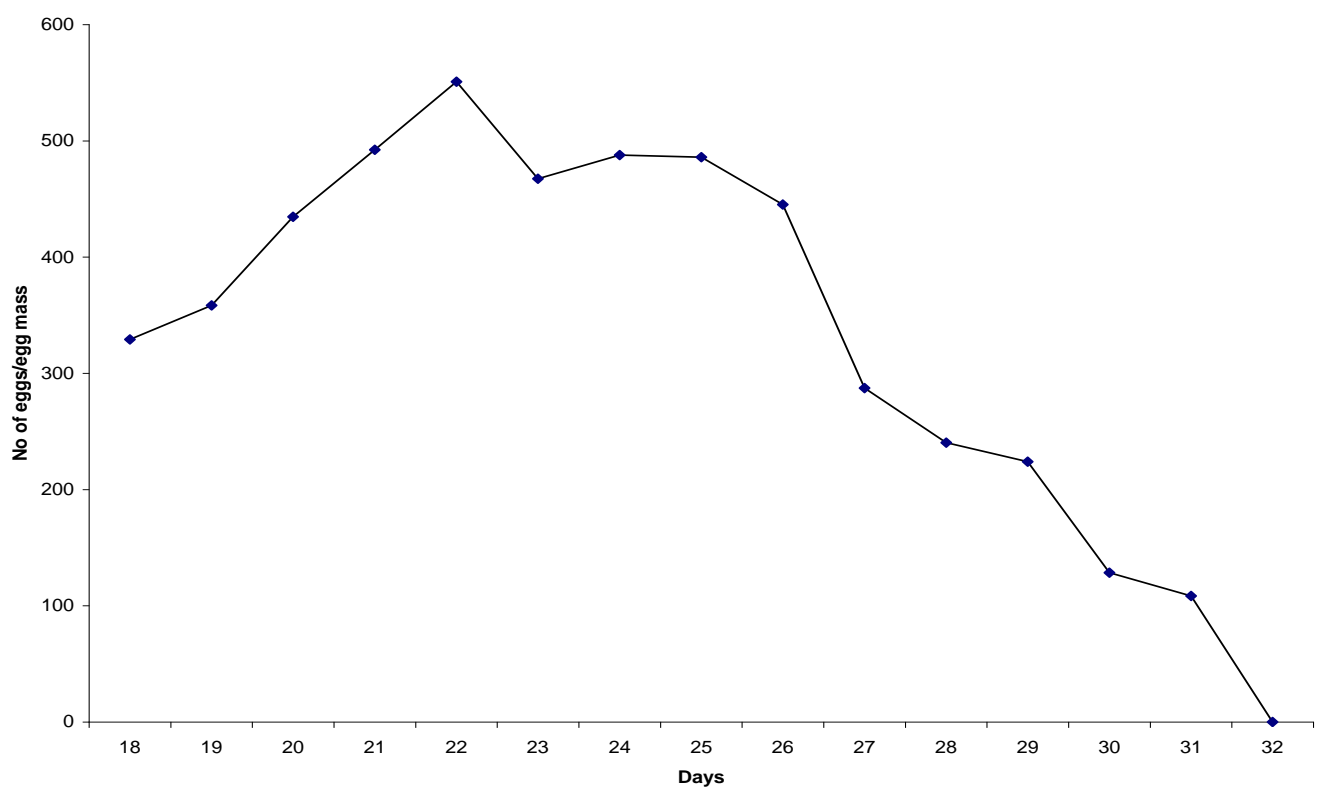

Fig.2 Age specific fecundity for healthy females

Population growth statistics of healthy and Pasteuria penetrans infected $M$. incognita

The study revealed that egg laying of healthy female starts from $18^{\text {th }}$ day but in $P$. penetrans infected $M$. incognita egg laying starts from $21^{\text {st }}$ day (Fig 1 and Fig 2). The fecundity of the $P$. penetrans infected females was very low. Some spores of $P$. pentrans attached to $M$. incognita juveniles failed to germinate. These juveniles produced eggs. The egg laying capacity of these females were partially affected (Fig 1). This was in accordance with Pembroke et al., (2004) who reported that some encumbered $\mathrm{J} 2$ which does not show spore germination has laid few eggs. 
Eggs per egg mass ranged from 90-148. Average eggs laid by the infected females were 80.3 eggs per female. The fecundity was reduced by $4-5$ times when compared to healthy $M$. incognita. This shows the parasite is highly efficient in arresting the fecundity of M. incognita significantly.

The decrease in innate capacity of $P$. penetrans infected root knot nematodes showed that the nematodes lost its ability for efficient multiplication compared to healthy nematodes. This leads to subsequent increase in doubling time of $M$. incognita infected by the $P$. penetrans. The rate of weekly multiplication of $M$. incognita population was lowered by 2.42 times (Table 8 and 9). The females developing inside the root was reduced significantly due to the spore burden on them (Davies et al., 1991).

\section{References}

Abad, P., Gouzy, J., Aury, JM., CastagnoneSereno, P and Danchin EG. 2008. Genome sequence of the metazoan plant-parasitic nematode Meloidogyne incognita. Nature Biotechnology 26: 909-915.

Atwal, AS., and Bains SS. 1974. Life Table studies In Applied animal ecology. Kalyani Publishers, India. 11-35pp.

Bansa, S., and Dhawan SC. 1994. Effect of Pasteuria penetrans on the penetration and multiplication of Heterodera cajani in Vigna unguiculata roots. Nematologia Mediterranea 22: 159161.

Birch, LC., 1948. The intrinsic rate of increase of an insect population. Journal of Animal Ecology 17: 15-26.

Davies, KG., 2009. Understanding the Interaction Between an Obligate Hyperparasitic Bacterium, Pasteuria penetrans and its Obligate Plant Parasitic Nematode Host, Meloidogyne spp. Advances in Parasitology 68: 211245.

Davies, KG., Laird, V., and Kerry, BR. 1991. The motility development and infection of Meloidogyne incognita encumbered with spores of the obligate hyperparasite Pasteuria penetrans. Revue de Nematologie 14: 611-618.

Davies, KG., Rowe, J., Manzanilla Lopez, R., and Opperman, CH. 2011. Reevaluation of the life-cycle of the nematode-parasitic bacterium Pasteuria penetrans in root-knot nematodes, Meloidogyne spp. Nematology 13: 825835.

Davies, KG., Kerry, BR., and Flynn, CA. 1988. Observations on the pathogenicity of Pasteuria penetrans, a parasite of root-knot nematodes. Annals of Applied Biology 112: 491-501.

Deevey, E,. 1947. Life tables for natural populations of animals. Queensland Review of Biology 22: 283-314.

Ferrls, H., and Hunt, WA. 1979. Quantitative aspects of the development of Meloidogyne arenaria larvae in grapevine varieties and root stocks. Journal of Nematology 11: 168-174.

Giannakou, I., and Gowen, SR. 2004. Factors affecting biological control effectiveness of Pasteuria penetrans in Meloidogyne javanica and the bacterial development in the nematode body. Nematropica 34: 153-163.

Howe, RW., (1953). The rapid determination of intrinsic rate of increase of an insect population. Annals of Applied Biology 40: $134-155$.

Pembroke, B., Darban, DA., and Gowen, S. 2004. Pasteuria penetrans: a tritrophic interaction. Bulletin-OILB/SROP 27: 229-234.

Rangaswamy, SD., Reddy, PP., Gowda, DN., and Nagesh, M. 2001. Influence of Pasteuria penetrans Sayre and Starr on the life cycle of Meloidogyne 
incognita (Kofoid and White) Chitwood infecting tomato. Pest Management in Horticultural Ecosystems 7: 168-170.

Singh, M., and Sharma, SB. 1994. Temperature effects on development and reproduction of Heterodera cajani on pigeonpea. Journal of Nematology 26: 241-248.

Singh, M., and Sharma, SB. 1995. Life table for Heterodera cajani on pigeonpea
(Cajanus cajan) Journal of Nematology 18: 309 -313.

Southwood, TRE., 1976. Ecological methods with particular reference to the study of insect populations. The English language book society and chapman and hall Pasteuria penetrans, 524pp.

Stirling, GR., 1984. Biological control of $M$. Javanica with Pasteuria penetrans. Phytopathlogy 74: 55 -60.

\section{How to cite this article:}

Prabhu. S, J. Sandeep Kumar and Poorniammal. R. 2020. Biology and Life Table Studies on Healthy and Pasteuria penetrans Infected Meloidogyne incognita. Int.J.Curr.Microbiol.App.Sci. 9(03): 3008-3018. doi: https://doi.org/10.20546/ijcmas.2020.903.345 